\title{
VEGFA and ILI7 expression reveals their potential functional crosstalk in periodontitis rats-A new animal model for angiogenesis study
}

\begin{abstract}
Vascular Endothelial Growth Factor A (VEGFA) is a main player in physiological and pathological angiogenesis in cardiovascular development, organs remodeling, wound healing, cell infiltration in inflammation, tumor development and metastasis. Interleukin 17 (IL17) is pro-inflammation cytokine secreted by a variety of cell types playing an important role in progression of infection, cardiovascular disease, autoimmune disease, and diabetes. Despite the emerging reports show that VEGFA and IL17 are both involved in vasculogenetic diseases, there is an evident paucity of information about functional cross talk between these two cytokines. Here, we analyze expression pattern of VEGFA and IL17 in a new periodontitis rat model through immunohistochemistry (IHC) staining. A total of 110 jaw tissues is taken at designed time points from rats. Periodontitis is created by sticking an orthodontic square wire on the surface of the left maxillary first molar (Group A, $\mathrm{n}=35$ ), ligating a metal wire ligature on the edge of gingival sulcus of the left mandibular first molar (Group B, $\mathrm{n}=35$ ), or both an orthodontic square wire on the surface of the left maxillary and a ligature in the cervical of the left mandibular first molar are installed at the same time (Group C, $\mathrm{n}=35$ ). Untreated rats periodontia (Control group, $\mathrm{n}=5$ ) are used as normal control. Histological analysis confirms the inflammatory features developing through time in interferenced rats periodontal tissues. Pathological analysis of VEGFA and IL17 expression shows that VEGFA and IL17 positive cells increase overtime comparing to control group; VEGFA and IL17 positive polykaryon cells, pericytes and endothelial cells are found around immature vessels near and/or in alveolar bones; pathological vasculature changes in group A is greater than group B overtime, whereas bone resorption in group B is greater than group A; both development of vasculature and bone resorption are parallel in group $\mathrm{C}$, along with accompanying increase of both VEGFA and IL17 expression in stromal tissue, leukecytes, endothelial cells and pericytes around newly formed vessels, and osteoclast-like polykaryon cells; Extracellular IL17 expression is dominant and reaches peak at second week in group A, whereas intercellular IL17 expression is dominant in group B and reaches peak at forth week. Most interestingly, group C has dominant intercellular IL17 expression although extracellular IL17 reaches peak at second week same to group A. Removing of interferences reverses the increasing expression of VEGFA/IL17 in all three groups with milder inflammation. We conclude that a new animal model to study angiogenesis and bone destruction is well established by characterization of developing vasculature, infiltrated immune cells, periodontal ligament fiber disorder and alveolar bone destruction in this periodontitis model. The increase of VEGFA/IL17 expression in endothelial cells and pericytes around immature vessels, as well as osteoclasts-like polykaryons in alveolar lacunas in this rat model suggests that VEGFA and IL17 are probably involved in remodeling of bone and vasculature caused by inflammation through potential functional crosstalk. Inhibition of producing of VEGFA and/or IL17 may be a potential therapeutic strategy for cardiovascular diseases, tumor bone metastasis, and other immune diseases including neurodegenerative diseases and even leukemia.
\end{abstract}

Keywords: periodontitis rat model, VEGFA, IL17, angiogenesis, immune diseases, bone destruction
Volume I Issue 4 - 2014

\author{
Zhiyong Zhang,' Xiaojun Ge, 'Wenxuan \\ Zheng, ${ }^{2}$ Huizhen Chen,' Xin Wang ${ }^{3}$ \\ 'Department of Stomatology, Second Hospital of HEBEl Medical \\ University, China \\ ${ }^{2}$ Harrison International Peace Hospital, China \\ ${ }^{3}$ Acure Biotechnology, Inc., USA
}

Correspondence: Xin Wang, Acure Biotechnology, Inc., Ellicott City, Maryland, USA, Email xwang354@gmail.com

Received: May 01, 2014 | Published: July 3I, 2014
Abbreviations: VEGFA, vascular endothelial growth factor A; IL17, Interleukin 17; IHC, immuno histo chemistry; HIF, hypoxia inducible factor.

\section{Introduction}

Periodontitis, a common infectious disease in dental care is characterized by chronic inflammation and irreversible periodontium destruction, including loss of connective tissue adherent to relevant tooth and bone caused by gingival recession and alveolar bone destruction. ${ }^{1}$ Although the pathological progress of periodontitis is observed in the tooth and nearby tissue, there are more risk factors through entire body, including stress (physical stress like occlusal trauma, and mental stress), tobacco and/or alcohol consumption, medical conditions, especially patients who having diabetes mellitus. ${ }^{2}$ Bite force is essential for normal physiological metabolic homeostasis 
of periodontal tissue to maintain normal jaw structure. However, the overloading of biomechanics, such as hyperocclusal force is harmful and causes destruction of connective tissue and bone associated with the teeth. ${ }^{3}$ More and more dentists intend to apply orthodontia into the therapeutic strategy after basic treatment of Periodontitis. ${ }^{4}$ The establishment of animal model of periodontitis combining occlusal trauma profoundly benefits to studying of the mechanism of destruction caused by inflammation and occlusal trauma, provides practical evidence for improving the effects of treatment for Periodontitis. ${ }^{5}$

It has been well established that Vascular Endothelial Growth Factor A (VEGFA) is an important cytokine for normal physiological and pathological angiogenesis in wound healing, inflammation, and tumor metastasis by inducing proliferation, inhibiting apoptosis of endothelial cells in newly formed vessels, thereby up-regulating the permeability of vascular. ${ }^{6}$ In 1998, VEGF was first time detected in endothelial cells, neutrophils, fibroblasts in periodontal tissue, epithelial cells in junction, pocket of gingiva in periodontitis patients. Since then, the emerging evidence showed that increasing VEGF expression is in keratinocytes of basal and spinous layers and de novo capillaries of type 2 diabetes associated gingiva over growth, epithelial and endothelial cells of periodontitis associated gingival., VEGF gene expression was also observed in pericytes contacting immature microvessels in developing retinal vasculature. ${ }^{10}$ However, whether VEGF produced by pericytes is involved in permeability of vessels is still in debate. Zhou et al., ${ }^{11}$ reported that VEGF blockade retains the association between pericytes and vessels forming in tumor xenografts derived from metastatic head and neck tumor cell lines although VEGF is well-known major hypoxia-inducible factor (HIF) controled gene in tumor angiogenesis and metastasis. ${ }^{12}$ However, our previous study showed that in aggressive head and neck cells, actin cytoskeleton-basic structure in cell migration is reorganizated, there by enhances migration of tumor cells under hypoxic condition. ${ }^{13}$ Location of pericytes in early phase of vessels is related to its separated VE-cadherin, a marker for leakiness of vessels, and actin rearrangement. ${ }^{11,14}$ Smooth muscle cells, another type of pericytes in prostate glands, have been shown more breaks in intracellular junctions in a transgenic mouse of androgen receptor (AR) short isform after castration..$^{15}$ Therefore, it is urged to develop an animal model for deciphering the function of VEGF produced by pericytes in tumor angiogenesis and metastasis caused by tumor microenvironment hypoxia and hormone deprivation.

IL17 is a pivotal cytokine playing a key role in strengthening immune response through improving secretion of chemokine, other cytokines to response against extracellular and intracellular pathogens by increasing granulopoiesis and recruiting leukocytes to the site of inflammation in the progression of infection. ${ }^{16} \mathrm{~A}$ convincing body of evidence has pointed that IL17 plays an important role in the pathophysiology of vascular inflammation and arteriosclerosis, ${ }^{17,18}$ and associated with autoimmune diseases, cardiovascular diseases and diabetes. ${ }^{19}$ Clinical research provided evidence linking major IL17 producing cell, Th17 cells to immune protection in leukemia, probably through promoting differentiation of this Thelper cell. ${ }^{20}$ Sato et al. ${ }^{21}$ demonstrated that IL17 expression affects the differentiation of osetoclasts, is crucial for $\mathrm{T}$ cell activity linked to bone resorption in a Th17 null autoimmune arthritis mouse model. In a clinical study with chronic periodontitis patients, Th17 expression significantly increases in gum tissue comparing to healthy gum. ${ }^{22}$ Recently, Silva and her colleagues found in a ligature-induced periodontal disease (PD) animal model combining with streptozotocin-induced type 1 Diabetes Mellitus (DM1) that IL23/IL17 expression associates with progression of PD in DM1, and increasing expression of Matrix metalloproteinases (MMPs) parralled with the tissues destruction. ${ }^{23}$

Here, we generate a new periodontitis rat model combining ligature wire stimulation with occlusal trauma for study the mechanism of VEGFA and IL17 involved vasculature and bone resorption caused by acute/chronic inflammation. The similar pattern of expression of VEGFA and IL17 reveals their potential function crosstalking in tissue destruction and remodeling. This is a new animal model for angiogenesis study.

\section{Materials and methods}

\section{Animal handling}

Three months old male Wistar rats, weight 250-280g, are purchased from Experimental Animal Center of Hebei Medical University (Shijiazhuang, China). 110 rats are randomly assigned into one control group (5 rats), and three experimental groups (35 rats each group). After being anesthetized by intraperitoneal injection of Chloral hydrate $(100 \mathrm{~g} / \mathrm{L}, \mathrm{H} 37022673$, Qingdao Yulong Algae Co.LTD, China) $(3 \mathrm{ml} / \mathrm{kg})$, and disinfected with $1 \%$ iodine tincture and $75 \%$ Alcohol (H20056160, Nanchang Baiyun Pharmacy Co. LTD, China), each rat of group A is ligated with a metal wire ligature (diameter 0.2mm, Grikin Advanced Materials CO., LTD, China) on the cervical of the left mandibular first molar, made the wire inside of the gingival sulcus each rat of group B is created the occlusal trauma by sticking an orthodontic square wire (diameter $0.6 \mathrm{~mm}$, Grikin Advanced Materials CO., LTD, China), length 1-2mm, on the grinded occlusal surface of the left maxillary first molar (Figures 1(A \& B)). The occlusal surface of the left maxillary first molar of Group B were raised $0.5-0.8 \mathrm{~mm}$ after the procedure; each rat in group $\mathrm{C}$ is installed both an orthodontic square wire on the surface of the left maxillary first molar and a ligature in the cervical of the left mandibular first molar at the same time. The rats in control group are received no any interfering. All rats are housed in pathogen-free cages at $25^{\circ} \mathrm{C}$ and $60-$ $70 \%$ humidity, fed a commercial diet.

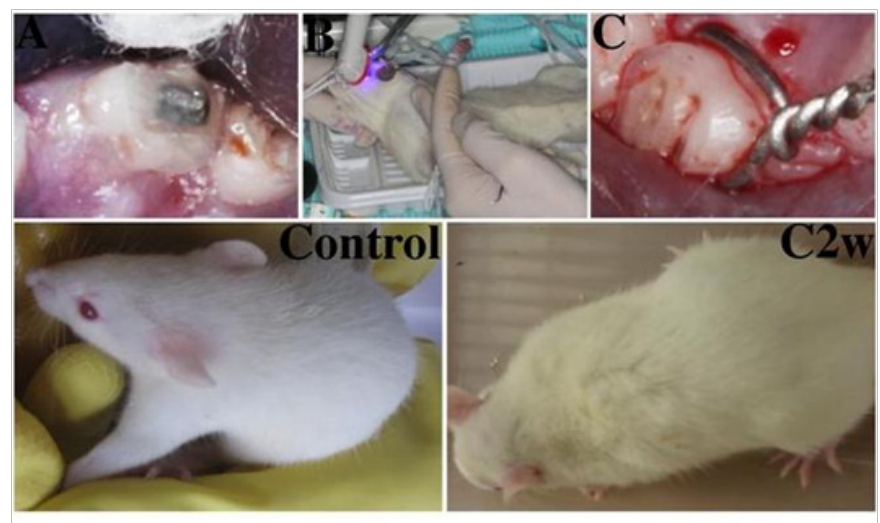

Figure I Establishment of rat models for creating periodontitis. The sticking of C-shape orthodontic square on the surface of first molar for occusal trauma rat model-Group $A(A)$; The operation of ligating a metal wire on first molar, and put it in gingival sulcus for interfering periodontitis rat modelGroup B(B); The sticking of C-shape orthodontic square on the surface of left maxillary first molar, whereas ligating a metal wire on mandibular first molarGroup C(C); A normal rat (Control); A rat model in Group C after installing materials 2 weeks.

At the first day, third day, seventh day, 2nd week, 4th week, respectively, 5 rats from each group are randomly chosen to euthanatizeby overdosed intraperitoneal injection of $10 \%$ Chloral 
hydrate $(4 \mathrm{ml} / \mathrm{kg})$, performed the transcardiac perfusion with $4 \%$ paraformaldehyde $(500 \mathrm{ml}$, Baihao Reagent CO. LTD, China), dissected for periodontal tissue including the left maxillary and mandibular molar area. 5 rats of each group are removed the interfering materials at 4th week under anesthetized situation, euthanatized at 6 th week with the left 5 rats in anesthetization for periodontal tissue. All animal experimental procedures are approved by the Office of Scientific Research Ethic Management of Second Hospital, Hebei Medical University (permit number: 2012L-3) and performed in accordance with the guideline from National Committee of Animal Care and Use in the animal experiments. All efforts are made for minimize animal suffering.

\section{Histopathological analysis}

The Periodontal tissues are fixed in 4\% paraformaldehyde for 48 hours, and decalcified in 10\% EDTA (Dentsply, USA) for 4 weeks, then embedded in wax and sectioned in mid-sagittal directionat a thickness of approximately $5 \mu \mathrm{m}$. Hematoxylin-eosin (HE) staining is performed following the standard protocol.

\section{Immunohistochemical staining}

Staining is performed according to the instructions of IHC. Briefly, after unmasked with $10 \mathrm{Mm}$ citrate buffer (PH 6) and following with $0.2 \%$ TritonX100 in PBS, endogenous peroxidase activity is blocked with $6 \%$ hydrogen peroxide for $30 \mathrm{~min}$ at room temperature. The slides are washed and pretreated with universal blocking solution (Dako, Car-pinteria, USA). Slides are incubated overnight at $4^{\circ} \mathrm{C}$ using 1:200 diluted goat anti-rabbit VEGFA(R131) (BS2431, BIOWORLD TECHNOLOGY, Inc, USA) polyclonal antibodies, 1:80 diluted IL17 antibody(H-132) (sc-7927, Santa Cruz Biotechnology, USA). The normal goat IgG AB-108 -C (R\&D Systems, USA) is used as negative control for primary antibodies. The slides are rinsed and incubated with biotinylated secondary antibody for $30 \mathrm{~min}$ at room temperature. After washing in PBS, the complex Strepta-vidin/ HorseRadish Peroxidase (Vector, USA) is applied for $30 \mathrm{~min}$ at room temperature. The reaction result is visualized with $\mathrm{DAB} /$ hydrogen peroxide kit (Dako,Car-pinteria, USA). Slides are observed by using the Olympus BX51T-PHD-J11 microscopy. Image-Pro Plus 6.0 image analysis software is utilized for the qualification analysis.

\section{Results}

\section{Periodontitis rat models are created through interfe- rence procedure}

A metal wire ligature is ligated on the gingival sulcus of each rat in group A (Figure 1C). An orthodontic square wire is stuck on maxillary first molar of each rat after grinding occlusal surface to generate group B (Figure 1(A \& B)). Group C is created by installing both an orthodontic square wire on the surface of the left maxillary first molar and a ligature in the cervical of the left mandibular first molar at the same time. Two rats are used to replace two died rats each from group A and group B due to anesthesia. Rats in the control group exhibit smooth, glossy white hair in good mood function (Figure 1 Control), whereas rats in experimental groups appear messy, lightless yellowing hair (Figure $1 \mathrm{C} 2 \mathrm{w}$ ). The rats in experimental groups are in bad appetite after interference procedure until turning to 3 rd week. Rats in experimental groups look exhausted and exhibit decreased activities over time.

\section{Periodontitis features are confirmed through histolo- gy analysis of h\&e staining}

Coronary sections of periodontal jaws in control rats show that periodontal ligament fibers arearranged orderly. Apical area of periodontal ligament is adhered tightly to alveolar bone. The lines of demarcation between alveolar bone and root of teeth are clear. Bone resorption lacunas distribute evenly with regular size. Osteoclasts occasionally can be found around vessels inside of alveolar bone lacunas (Figure 2A).

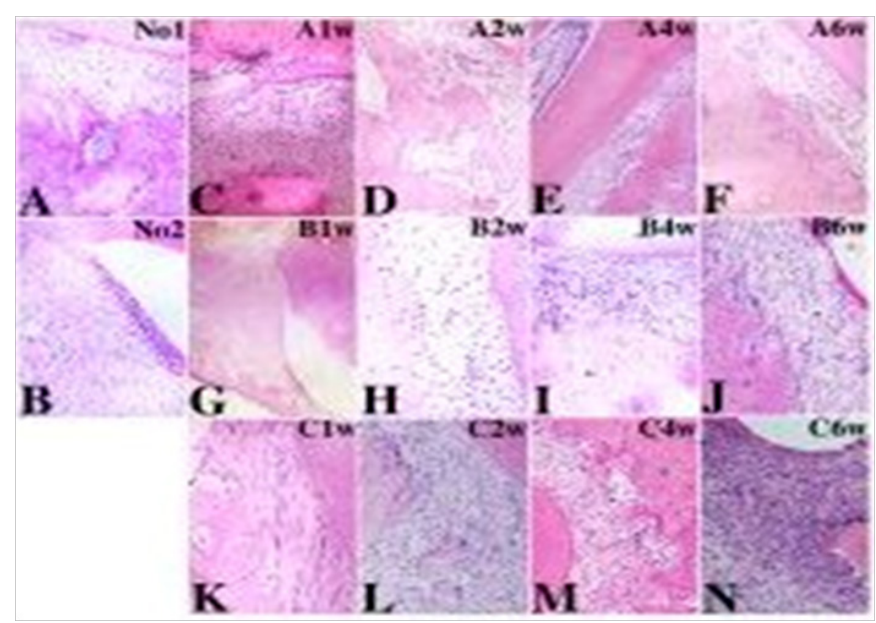

Figure 2 Histological analysis of periodontal tissue in periodontitis rat models. H\&E Staining was performed with gingival tissue taken from control group and experimental groups. A, Normal periodontal tissue (No I, normal I); B, The epithelial tissue normally adhered to root of tooth in control rat (No 2, magnificent200x); C, Periodontal ligament fibers slightly lost order at first week in group $\mathrm{A}(\mathrm{AI}$, I 100x); D, Blood vessel dilatation and congestion at second week in group $A(A 2 w, 100 x)$; E, Apical resorption of alveolar bone at 4th week in group A (A4w, 100x); F, Superficial newborn of alveolar bone seen at 6th week in group A (A6w, 200x); G, Normal epithelial tissue adherent on root of tooth at first week in group B (BIw, 200x); H, Occasionally osteoclast seen on the surface of alveolar bone at second week in group B (B2w, 200x); I, More osteoclast seen on the surface of alveolar bone at 4 th week in group B (B4w, 200x); J, The accumulation of osteoclast-like cells seen on the surface of alveolar bone at 6th week in group B (B6w, 400x); K, The blood vessel dilatation at first week in group $\mathrm{C}(\mathrm{Clw}, 200 \mathrm{x})$; L, Bone resorption at second week in group C (C2w, 100x); M, Areolar connective tissue replaced normal periodontal tissue at 4th week in group $C(C 4 w, 100 x) ; N, A$ lot of inflammation cell infiltrated around alveolar bone at 6th week in group $C$ (C6w, 100x). B, alveolar bone; D, dental tooth; OC, Osteoclast-like cells; P, periodontal ligament fibers; LCT, Loose connective tissue.

Comparing to control group, the rats in experimental groups show that increased fibroblasts and infiltrated immune cells in periodontal tissue and order-lost fibers in the periodontal ligament appear at first week, get serious from second week to the end time point sixth week (data not shown). At the second week of group A, periodontal ligament fibers slightly lost order with blood vessel dilatation, congestion, and apical resorption of alveolar bone with superficial newborn of alveolar bone (Figure 2B); Periodontal tissue of group B at 2nd week show that epithelial tissue lost adherent on root of tooth, periodontal ligament fibers lost order, osteoclasts-like polykaryons are seen on the surface of alveolar bone (Figure 2C); In group C, areola connective tissue replaced normal periodontal tissue, the blood vessel shows dilatation, bone resorptionis obvious, a lot of inflammation cell infiltrated around alveolar bone (Figure 2D). More and more osteoclasts- 
like polykaryons are found around vessels in the progressive bone resorption lacunas over time. The histological changes appear more obvious in the periodontitis rat model when combined with occusal trauma (data not shown). VEGFA expression increases overtime in stroma, leukucytes, endothelial cells and pericytes of newly formed vessels, osteoclasts-like polykaryons around vessels in progression of bone resorption lacunas. In control group, there are a few VEGFA positive osteoclasts are located near the large matured vessels inside of alveolar bone and periodontal ligament (Figure 3A); No VEGFA positive polymorphonuclear leukocytes are seen near the vessels in periodontal tissue (Figure 3B).

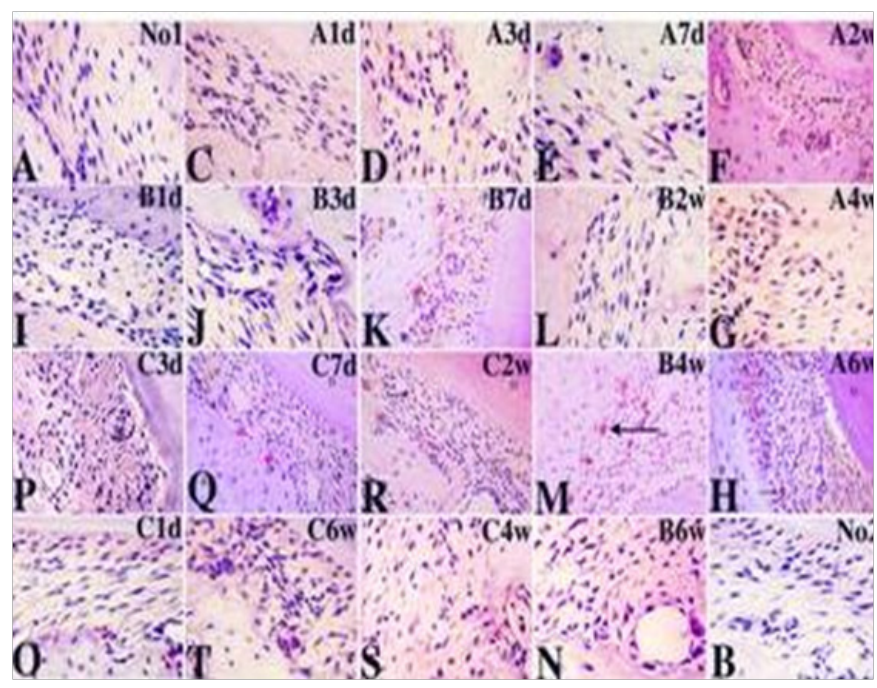

Figure 3 VEGF expression in Periodontal Tissue in Three Periodontitis Rat Models. Immuno histochemical analysis of VEGF A, No I, normal VEGF expression pattern in connective tissue between alveolar bone and cementum in control rats (400x); B, No 2, normal blood vessels and VEGF positive cells localized in periodontal tissue of control rats (400x); C, AId, a few VEGF positive fibroblasts existed in periodontal ligament that is in good order at first day in group A (400x); D, A3d, the weak VEGF positive endothelial cells were seen in alveolar bone; the periodontal ligament fibers lost order at third day in group A (400x); E, A7d, the osteoclasts increased along the surface of alveolar bone at seventh day in group A (400x); F, A2w, the number of VEGFA positive cells increased, and appeared intensive staining in alveolar bone lacunas at 2nd week in group A (200x); G, A4w, fibers in periodontal tissue were obviously disorder, the most positive cells localized on the surface of alveolar bone at 4th week in group $A(400 x) ; H, A 6 w$, the number of positive cell decreased, fibers in periodontal tissue lost order at 6 week in group A (200x); I, B Id, a few positive cells with weak VEGF expression were seen at first day in group B (200x); J, B3d, the osteoclasts were occasionally observed in lacunas at third day in group B $(400 x) ; \mathrm{K}, \mathrm{B} 7 \mathrm{~d}$, the endothelial cells appeared weak VEGF expression at seventh day in group B (200x); L, $B 2 w$, the number of positive osteoclasts increased at 2nd week in group B (400x); M, B4w, more intensive staining of VEGF of osteoclasts were found on the surface of alveolar bone at 4th week in group B (200x); N, B6w,VEGFA positive osteoclasts and dilated blood vessels were toward the alveolar bone surface at 6 th week in group $\mathrm{B}(400 \mathrm{x}) ; \mathrm{O}, \mathrm{CId}$, a few positive osteoclast-like cells had been seen along the surface of alveolar bone at first day in group C (400x); P, C3d, severer disorder of periodontal ligament had been seen at third day in group C (400x); Q, C7d, the number of lacunas and osteoclast-like cells increased at seventh day in group C (200x); R, C2w, the number of blood vessel in periodontal tissue increased, osteoclast-like cells multinucleated with positive VEGF expression at 2nd week in group C (200x); S, C4w, fibers of periodontal tissue obviously disordered at 4 th week in group $C(400 x) ; T, C 6 w$, more lacunas were observed, the VEGF positive cells accumulated at 6 th week in group $\mathrm{C}(400 \mathrm{x})$. $\mathrm{B}$, alveolar bone; $\mathrm{P}$, periodontal ligament; $\mathrm{D}$, dental tooth; White Triangles indicate VEGF positive osteoclast-like cells having polynuclei; Arrows show the VEGF positive vessels.
Distribution of VEGFA positive cells have some characters in experimental rats models of periodontitis (Figures 3(A, C-T)): First, in experimental groups, number of VEGFA positive vessels and VEGFA positive cells increase since first day interfered by dental materials, progressively increase over the observing periods. Second, VEGFA positive leukucytes, endothelial cells and pericytes are found near newly formed vessels, or formed the structure of immature vessels. Osteoclasts-like polykaryons are found in bone resorption lacunas and apical area of root of all molars containing first, second and third molar. Third, experimental groups appear different observable period of multilobulated nuclei cells: in Group A, VEGFA positive polykaryons are observed from 7th day; in Group B, are observed since 3rd day; in Group C, are observed as early as first day. Forth, the frequentation of number of VGEFA positive microvessels and VEGFA positive cells in bone destruction is: control group, group B, group A, group C. VEGFA positive newly formed vessels concentrate in apical area of alveolar bone are seen in periodontal tissue. Comparing to control group, the pathological changes of VEGFA expression in group $\mathrm{C}$ is greater than group $\mathrm{B}$ that is greater than group $\mathrm{A}$.

\section{ILI 7 expression pattern is very similar to VEGFA}

Similar to VEGFA, IL17 expression increases in stroma tissue, inflammation cells, endothelial cells and pericytes around microvessels, and osteoclast-like polykaryons in bone resorption lacunas with the progression of periodontal inflammation (Figure 4), and IL17 positive leukucytes and pericytes start to appear at the first day in all three experimental groups(Figures 4(A1d, B1d \& C1d). In Group A, microenvironmental IL17 expression is most dominant, reaches the peak at $2 \mathrm{w}$ (Figure $4 \mathrm{~A} 2 \mathrm{w}$ ). In group B, intercellular IL17 expression is dominant(Figures $4(\mathrm{~B} 1 \mathrm{~d}-\mathrm{B} 6 \mathrm{w})$ ), microenvironmental IL17 expression reaches the peak at $4 \mathrm{w}$ (Figure 4B4w). Most interestingly, Group C has most dominant intercellular IL17 expression although microenvironmental IL17 reaches the peak at $2 \mathrm{w}$ same to Group A (Figures (4C1d-C6w)). Removing of interference reverses the increasing expression of IL17 in all three groups (Figures 4(Ar, $\mathrm{Br} \& \mathrm{Cr}$ )). There are earlier microvessels formed marked by IL17 expression in group A than group B, whereas bone resorption marked by IL17 positive osteoclast-like polykaryons in group B occurred earlier and greater than group A, and development of vasculature and bone resorption with stronger IL17 expression profoundly paralleled in group $\mathrm{C}$.

\section{Discussion}

Both VEGFA and IL17 had been reported key cytokines in cardiovascular diseases, acute and chronic inflammation, immune and autoimmune diseases, and physiology or pathology tissue and bone remodeling. VEGF is involved in angiogenesis by promoting differentiation of mesenchymal stem cells toward endothelial cells, ${ }^{11}$ whereas IL17 is reported that involved in immune response by promoting differentiation of blood stem cell (peripheral and bone marrow) toward T help cells and endothelial cells in angiogenesis. ${ }^{24}$ However, whether there is potential functional crosstalk between these two cytokines remains unknown. Our study addressed this question, first time revealed the similar expression pattern of VEGF and IL17 in acute, and chronic inflammation in a new periodontitis rat model, thereby provided supportive evidence of functional crosstalk between these two proteins.

The mechanism that involved in angiogenesis and bone destructive pathologic process has been reported that associated with deadly disease like cancer metastasis to bone. ${ }^{25}$ On the other hand, the bone 
destruction caused by chronic inflammation disease like rheumatoid arthritis, vascular disease, periodontitis; and metabolic abnormal like diabetes; and aging and hormone disorder, all seriously affect the quality of daily life of patients. A study tool is needed for study the progression of these disease and therapeutic strategies in reducing bone resorption and tumor metastasis, prevent future disability. ${ }^{26} \mathrm{We}$ successfully establish periodontitis rat model in this study.

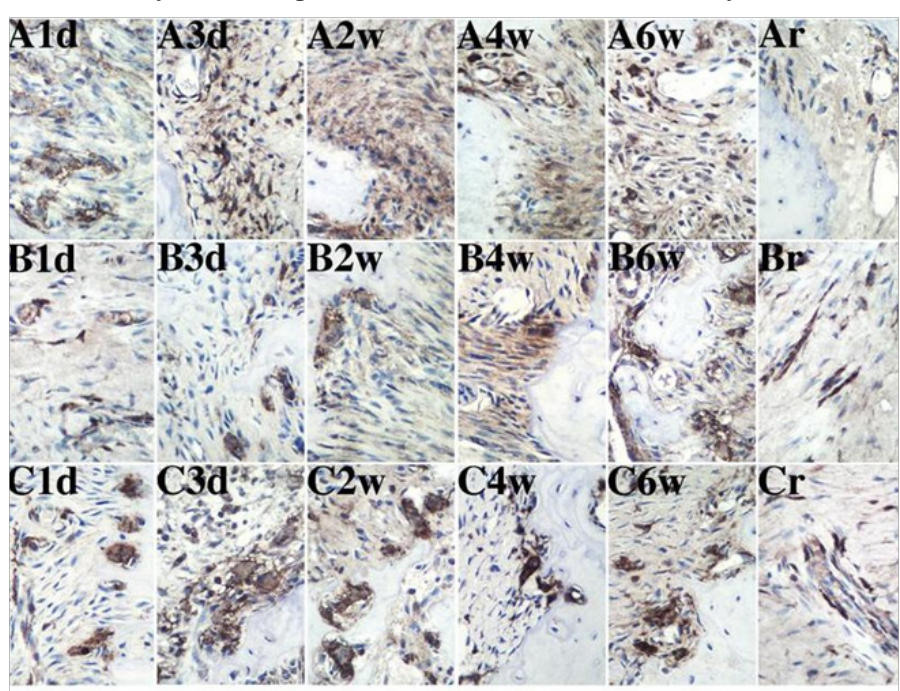

\section{anti-IL17 400x}

Figure 4 ILI7 Expression Pattern in Periodontitis Rat Model. "A" indicates Group A. Microenvironment dominant ILI7 expression increases at the first day in Group $A(A I d)$, through 3rd day $(A 3 d)$, reach the peak in cell micro environment at 2 weeks ( $A 2 w)$, micro environmental expression of ILI7 starts reducing at 4 weeks and 6 weeks (A4w, A6w), whereas intercellular expression of ILI7 still remain high level; "B” indicates Group B. Expression of ILI7 increases from first day (BId); through 3rd day (B3d), 2 weeks (B2w), reach the peak at 4 weeks in microenvironment (B4w), reduces at 6 week (B6w) whereas intercellular expression of ILI7 is still high; "C" indicates Group C. Intercellular expression of ILI7 greatly increases at first day (CId) in osteoclast-like polykaryon cells along the surface of alveolar bone, and upregulate over of the time, 3rd day (C3d), 2 weeks (C2w), 4 weeks $(C 4 w)$, to endpoint 6 weeks ( $66 \mathrm{w})$. 2 weeks after removing interfered orthodontic square, and/or wire $(\mathrm{Ar}, \mathrm{Br}, \mathrm{Cr})$ at 4 weeks, expression of ILI 7 reverses in both intercellular and microenvironment in all three groups. Intercellular expression of ILI7 is the highest in Group C among three models, whereas micro environmental expression of IL 17 is the highest in Group A, Group B has the lowest expression of ILI 7in both intercellular and microenvironment among three groups. Magnificent is $400 x$.

Traditionally, VEGF family is a group of proteins playing important role in angiogenesis in normal development, wound healing, acute and chronic inflammation, and tumor growth. Emerging evidence suggest that VEGF involved in osteogenesis because VEGF and its receptors present on osteoblasts and osteoclasts. ${ }^{27}$ Our study provides supportive data that VEGF express not only in endothelial cells of new neovascular vessels, but also in pericytes of immature microvessels and polynucleat osteoclast-like cells in enlarged alveolar lacunas. IL17 expression appear similar pattern. This suggests that VEGF and IL17 both may be essential molecules for neovasculature during osteogenesis. The often used model for coupling of osteogenesis and angiogenesis is autoimmune arthritis. ${ }^{28}$ This study provides a new feasible practical tool for study the mechanism of coupling of osteogenesis and angiogenesis.
A single nucletotide gene polymorphisms (SNPs) shows that periodontitis patients more frequently appear CC genotype and $\mathrm{C}$ allele of VEGF. ${ }^{29}$ This suggests that epigenetic modification may play roles in regulation of VEGF expression and function in progression of periodontitis. Moreover, it has been report that higher level of VEGF concentration in both gingiva crevicular fluid and serum from periodontitis patients is related to the disease's severity. ${ }^{30}$ The evidence above suggests that VEGFA is an important molecule in progression of periodontitis, and its expression is closely associated with periodontal tissue destruction. However, the neovascularization mechanism that VEGF involved remains to be precisely demonstrated.

\section{T helper(Th I 7) cells producing IL-I 7 playing a crucial role in autoimmune}

inflammation has just been recognized. It has been indicated that Th17 cells is an osteclastogenic subset that closely associated with bone damage in autoimmune disease in a RA model. ${ }^{31}$ We obtain similar results in this new periodontitis rat model. Supprisingly, the IL17 expression patterns are different between hard metal wire caused inflammation and occlusive trauma caused inflammation. There is more secreted IL17 in periodontal stroma microenvironment in occlusive trauma periodontitis model, which may indicate that inflammation processing signaling is different between mechanery and fractionary damage. When both interfering exist, the consequence of inflection is the worst. Our previous study discovered a novel gene (BAALC) that has active $\mathrm{N}$-terminal lipids raft targeting motif similar to G-protein family. ${ }^{32}$ It highly expressed in blood cells of leukemia patients, and associated with differentiation of blood stem cells, ${ }^{33,34}$ which overlapped with function of IL17. Targeting IL17 may provide new strategy for therapy of leukemia.

\section{Conclusion}

New Rat periodontitis model for study the mechanism of angiogenesis and bone destruction has been established. The expression of VEGF and IL17 in the periodontal tissue appears very similar, indicate that both are involved in periodontal tissue injury and repair process through potential functional crosstalk. Inhibition of producing of VEGFA and/or IL17 may be a potential therapeutic strategy for cardiovascular diseases, tumor bone metastasis, and other immune diseases including neurodegenerative diseases and leukemia.

\section{Acknowledgments}

None.

\section{Conflicts of interest}

Authors declare that there is no conflict of interest.

\section{References}

1. Ara T, Kurata K, Hirai K, et al. Human gingival fibroblasts are critical in sustaining inflammation in periodontal disease. $J$ Periodontal Res. 2009;44(1):21-27

2. Page RC, Beck JD. Risk assessment for periodontal diseases. Int Dent J. 1997;47(2):61-87

3. Nokhbehsaim M, Deschner B, Winter J, et al. Contribution of orthodontic load to inflammation-mediated periated periodontal destruction. J Orofac Orthop. 2010;71(6):390-402.

4. Foz AM, Artese HP, Horliana AC, et al. Occlusal adjustment associated with periodontal therapy-a systematic review. J Dent. 2012;40(12):1025-1035. 
5. Sbordone L, Bortolaia C. Periodontal disease and occlusal trauma: a still debated controversy? A review of the literature. Minerva Stromatol. 2002;51(3):79-85.

6. Herr D, Bekes I, Wulff C. Regulation of Endothelial Permeability in the Corpus Luteum: A review of the literature. Geburtshilfe Frauenheilkd. 2013;73(11):1107-1111.

7. Booth V, Young S, Cruchley A, et al. Vascular endothelial growth factor in human periodontal disease. J Periodontal Res. 1998;33(8):491-499.

8. Morelli T, Neiva R, Nevins ML, et al. Angiogenic biomarkers and healing of living cellular constructs. J Dent Res. 2011;90(4):456-462.

9. Aspriello SD, Zizzi A, Lucarini G, et al. Vascular endothelial growth factor and microvessel density in periodontitis patients with and without diabetes. J Periodontol. 2009;80(11):1783-1789.

10. Darland DC, Massingham LJ, Smith SR, et al. Pericyte production of cell-associated VEGF is differentiation-dependent and is associated with endothelial survival. Dev Biol. 2003;264(1):275-288.

11. Zhou H, Yang YH, Basile JR. The semaphorin 4D-Plexin-B1-RhoA signaling axis recruits pericytes and regulates vascular permeability through endothelial production of PDGF-B and ANGPTL4. Angiogenesis. 2014;17(1):261-274.

12. Semenza GL. Regulation of hypoxia-induced angiogenesis: a chaperone escorts VEGF to the dance. J Clin Invest. 2001;108(1): 39-40.

13. Wang X, Schneider A. HIF-2alpha-mediated activation of the epiderma growth factor receptor potentiates head and nech cencer cell migration in response to hypoxia. Carcinogenesis. 2010;31(7):1202-1210.

14. Yuan D, He P. Vascular remodeling alters adhesion protein and cytoskeleton reactions to inflammatory stimuli resulting in enhanced permeability increases in rat venules. J Appl Physiol. 2012;113(7):1110-1120.

15. Sun F, Chen HG, Li W, et al. Androgen receptor splice variant AR3 promotes prostate cancer via modulating expression of autocrine/ paracrine factors. J Biol Chem. 2014;289(3):1529-1539.

16. Ouyang W, Kolls JK, Zheng Y. The biological functions of T helper 17 cell effector cytokines in inflammation. Immunity. 2008;28(4):454- 467.

17. von Vietinghoff S, Ley K. Interleukin 17 in vascular inflammation Cytokine Growth Factor Rev. 2010;21(6):463-469.

18. Smith E, Prasad KMR, Rutcher M, et al. Blockade of interleukin-17A results in reduced atherosclerosis in apolipoprotein E-deficient mice. Circulation. 2010;121(15):1746-1755.

19. Kramer JM, Gaffen SL. Interleukin-17: a new paradigm in inflammation, autoimmunity, and therapy. J Periodontol. 2007;78(6):1083-1093.

20. Abousamra NK, El-Din MS, Helal R. Prognostic value of Th17 cells in acute leukemia. Med Oncol. 2013;30(4):732-738.

21. Sato K, Suematsu A, Okamoto K, et al. Th17 functions as an osteoclastogenic helper T cell subset that links T cell activation and bone destruction. J Exp Med. 2006;203(12):2673-2682.
22. Behfarnia P, Birang R, Andalib AR, et al. Comparative Evaluation of IFN $\gamma$, IL4 and IL17 cytokines in healthy gingival and moderate to advanced chronic periodontitis. Dent Res J (Isfahan). 2010;7(2):45-50.

23. Silva JA, Ferrucci DL, Peroni LA, et al. Sequential IL23 and IL17 and increased Mmp8 and Mmp14 expression characterize the progression of an experimental model of periodontal disease in type 1 diabetes. $J$ Cell Physiol. 2012;227(6):2441-2450.

24. Kang J, Hur J, Kang JA, et al. Activated platelet supernatant can augment the angiogenic potential of human peripheral blood stem cells mobilized from bone marrow by G-CSF. J Mol Cell Cardiol. 2014;75:64-75.

25. Das Roy L, Pathangey LB, Tinder TL, et al. Breast-cancer-associated metastasis is significantly increased in a model of autoimmune arthritis. Breast Cancer Res. 2009;11(4):R56.

26. Pisoschi C, Stanciulescu C, Banita M. Growth factors and connective tissue homeostasis in periodontal disease. In: Buduneli $\mathrm{N}$, editor. Pathogenesis and Treatment of periodontitis. InTech: Croatia; 2002.

27. Uchida S, Sakai A, Kudo H, et al. Vescular endothelial growth factor is expressed along with its receptors during the healing process of bone and bone marrow after drill-hole injury in rats. Bone. 2003;32:491-501.

28. Kusumbe AP, Ramasamy SK, Adams RH. Coupling of angiogenesis and osteogenesis by a specific vessel subtype in bone. Nature. 2014;507(7492):323-328.

29. Loo WT, Wang M, Jin LJ, et al. Association of matrix metalloproteinase (MMP-1, MMP-3 and MMP-9) and cyclooxygenase-2 gene polymorphisms and their proteins with chronic periodontitis. Arch Oral Biol. 2011;56(10):1081-1090.

30. Sakallioglu EE, Aliyev E, Lutfioglu M, et al. Vascular endothelial growth factor (VEGF) levels of gingival and gingival crevicular fluid in diabetic and systemically healthy periodontitis patients. Clin Oral Investig. 2007;11(2):115-120

31. Van Bezooiejen RL, Van Der Wee-Pals L, Papapoulos SE, et al Interleukin 17 synergise with tumor necrosis factor alpha to induce cartilage destruction in vitro. Ann Rheum Dis. 2002;61(10):870-876.

32. Wang $\mathrm{X}$, Tian QB, Okano A, et al. BAALC 1-6-8 protein is targeted to postsynaptic lipid rafts by its $\mathrm{N}$-terminal myristoylation and palmitoylation, and interacts with alpha, but not beta, subunit of $\mathrm{Ca}$ /calmodulin-dependent protein kinase II. $J$ Neurochem. 2005;92(3):647-659.

33. Baldus CD, Tanner SM, Kusewitt DF, et al. BAALC, a novel marker of human hematopoietic progenitor cells. Exp Hematol. 2003;31(11):1051-1056

34. Tallman MS, Gilliland DG, Rowe JM. Drug therapy for acute myeloid leukemia. Blood. 2005;106(7):1154-1163. 